International Journal of Bioinformatics Research

ISSN: 0975-3087, E-ISSN: 0975-9115, Vol. 3, Issue 1, 2011, pp-185-189

Available online at http://www.bioinfo.in/contents.php?id=21

\title{
INHIBITING H5N1 HAEMAGGLUTININ WITH SMALL MOLECULE LIGANDS
}

\author{
ADITI GANGOPADHYAY, SAYAK GANGULI AND ABHIJIT DATTA \\ DBT Centre for Bioinformatics, Presidency University, Kolkata \\ *Corresponding author. E-mail: sayakbif@yahoo.com / 09433081029
}

Received: January 10, 2011; Accepted: February 23, 2011

\begin{abstract}
Mutations in the avian influenza virus can create new pathogenic strains, which can cause future pandemics. Targeting the surface haemagglutinin can inhibit viral fusion and consequent entry. The effects of 27 single and double point mutations on the properties of the $\mathrm{H} 5$ haemagglutinin were studied. These mutations were then introduced individually in the PDB file of the $\mathrm{H} 5$, to generate mutants. 13 herbal and non-herbal lead inhibitors of $\mathrm{H} 5$ were screened and docked with the wild type and mutant haemagglutinins to obtain a comparative docking profile. The nature of the interactions of the inhibitors were analysed, and the binding residues determined. The latter further revealed that certain mutations in $\mathrm{HA}$ might be affecting inhibitor binding.

Keywords: H5N1 inhibitors, effect of H5 mutations,
\end{abstract}

\section{INTRODUCTION}

The creation of new avian influenza pandemics, and far more virulent strains of the virus are continually threatening the world (WHO, 2004). Such new strains arise via reassortment during a co-infection of the same host (Zhou et al, 1999), or by a gradual process of adaptive mutation (Horimoto and Kawaoka, 2001). The influenza surface haemagglutinin spike is a glycoprotein projecting from the surface of the virus. The globular head of this spike bears the receptor binding site, that binds to the host's sialic acid residues (Voet and Voet, 2004). Since the influenza surface haemagglutinin is responsible for the initial binding (Voet and Voet, 2004), hence, inhibiting the surface HA can keep the chances of infection and outbreak, at bay. Herbal, fatty acid and other chemical inhibitors against the $\mathrm{H} 5$ haemagglutinin were screened for their drug-likeliness.

\section{EFFECT OF MUTATIONS IN HAEMAGGLUTININ}

Single and double point mutations, which have been reported to alter various properties of the $\mathrm{H} 5$ haemagglutinin were studied, which vary in the range of altering the hydropathy index, to number of glycosylation sites, $\mathrm{pH}$ of activation, or even affinity of binding to the avian receptor. Some mutations promote avian to human transmission, while others at the antigenic site create 'escape mutants' which are able to escape the host's immune surveillance. An enhancement in the recognition and binding of the human receptors by the virus is also effected by some of these mutations. Some mutations are capable of altering the glycosylation sites on haemagglutinin. The importance of glycosylation can be traced to the fact that, the presence of carbohydrates on the haemagglutinin which are host specific, can mask surfaces of the haemagglutinin from the host's immune system, thus allowing it to escape unrecognised (Salahuddin and Khan, 2009). An interesting mutation, at 156, changing the usual alanine to serine or threonine, brings about the appearance of glycosylation sites in four strains of avian virus (Salahuddin and Khan, 2009). These two very mutations, however, brings about the disappearance of a glycosylation site in raccoon dog viruses (Qi et al., 2009). Haemagglutinin mutations at the antigenic sites, can produce strains that have acquired the ability of escaping binding by the antibody ((Salahuddin and Khan, 2009). The pathogenecity and transmissibility of the influenza viruses depends on the receptor specificity and clevability of the surface haemagglutinin. The cleavage of the precursor HAO subunit, into the two HA1 and HA2 fragments is a prior requisite for the haemagglutinin to be able to cause an effective fusion and consequent entry (Durrer et al., 1996). After binding to the sialic acid, the virus is engulfed into the endosome of increasingly low $\mathrm{pH}$. At a certain threshold $\mathrm{pH}$ (referred to as $\mathrm{pH}$ of fusion), the haemagglutinin undergoes a conformational change, that aids in the fusion of the virion with the endosomal membrane (Durrer et al., 1996; Reed et al., 2009). Certain mutations in the influenza can alter this $\mathrm{pH}$ of fusion, thus offering the virus a wider choice of host range and cell lines (Reed et al., 2009).

The alteration of the hydropathy index by mutations is an important source of pathogenesis (Salahuddin and Khan, 2009). Some of these mutations alter a hydrophilic residue to a hydrophobic one, which is 
believed to aid in the attainment of a stabler structure (Anwar et al., 2006).

\section{INHIBITORS SCREENED}

Docosanol has been suggested to have inhibitory activity against lipid-enveloped viruses (Katz et al., 1991), hence it has been used in the present study, as the influenza viruses are enveloped viruses. Chemical compounds having structural similarity with docosanol were next selected from DrugBank. The inhibitors may be classified into the herbal category, which are derived from plant sources and comprises of docosanol, eucalyptol, ormosinine and menthol; the saturated fatty acid inhibitors include caprylic acid, decanoic acid, lauric acid, palmitic acid, stearic acid. Dromostanolone is the only steroid inhibitor. Tranexamic acid is a cyclohexane carboxylic acid, while deoxycholic acid and ursodeoxycholic acid are bile acids.

\section{MATERIALS AND METHODS}

The PDB ID of the $\mathrm{H} 5$ avian haemagglutinin chosen for the present study is 1JSM. Ramachandran analysis using the MolProbity online server revealed that 1JSM contains 4 outliers (refer Figure 1: Supplementary). The 27 single and double point mutations (Table 1: Supplementary) were individually introduced in the $\mathrm{H} 5$ monomer of the PDB file 1JSM, using the Swiss PDB Viewer, version 4. 0.1 . Since the biologically active haemagglutinin is homotrimeric, hence, the wild type (1JSM) and mutant monomers were docked with each other, to form homotrimers.

The canonical SMILES of the inhibitors were obtained from DrugBank and PubChem, and their 3D structures were obtained using the Online SMILES translator and Structure file generator. Some of the 3D structures were obtained directly from DrugBank. These lead compounds were subjected to in silico screening for drug-likeliness, using the online Molinspiration virtual screening tool (refer Table 2: Supplementary, for screening results).

Next, the mutant and wild type (1JSM) trimers were docked with the lead inhibitors, using Hex 5.1 Docking software.

\section{RESULTS}

The results of the dockings are presented graphically, as under:

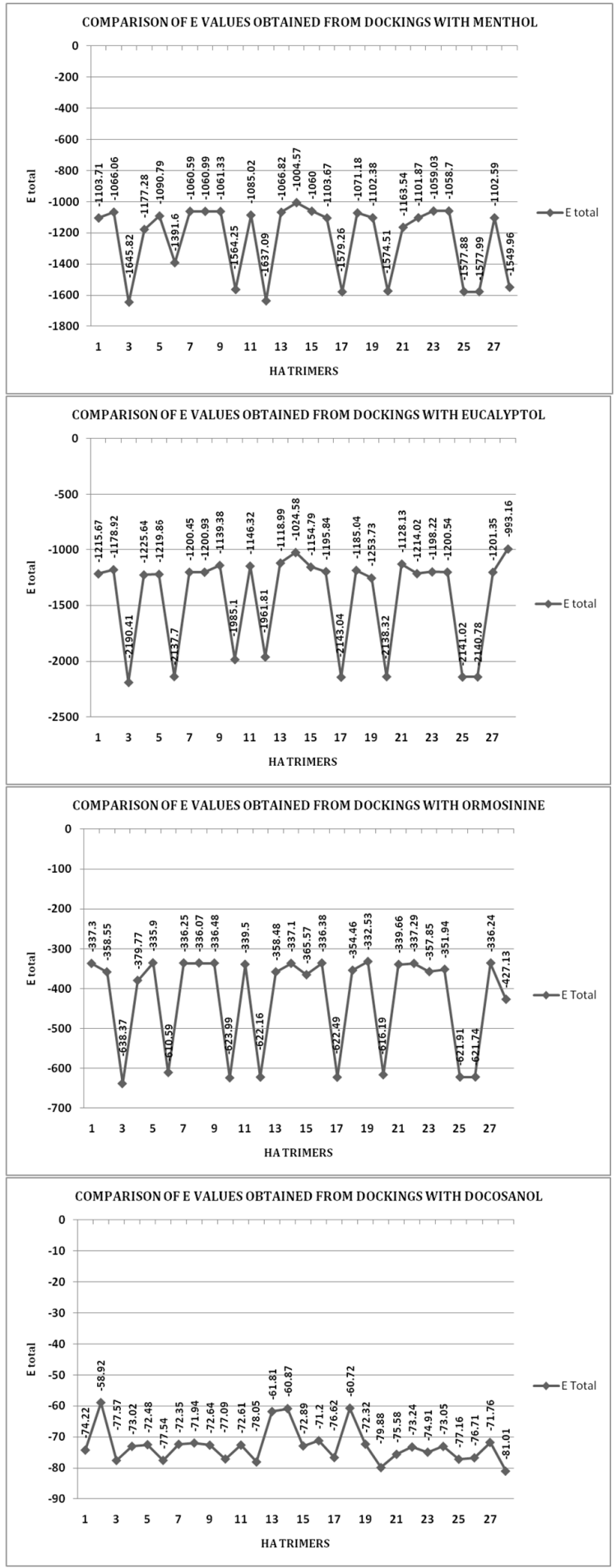



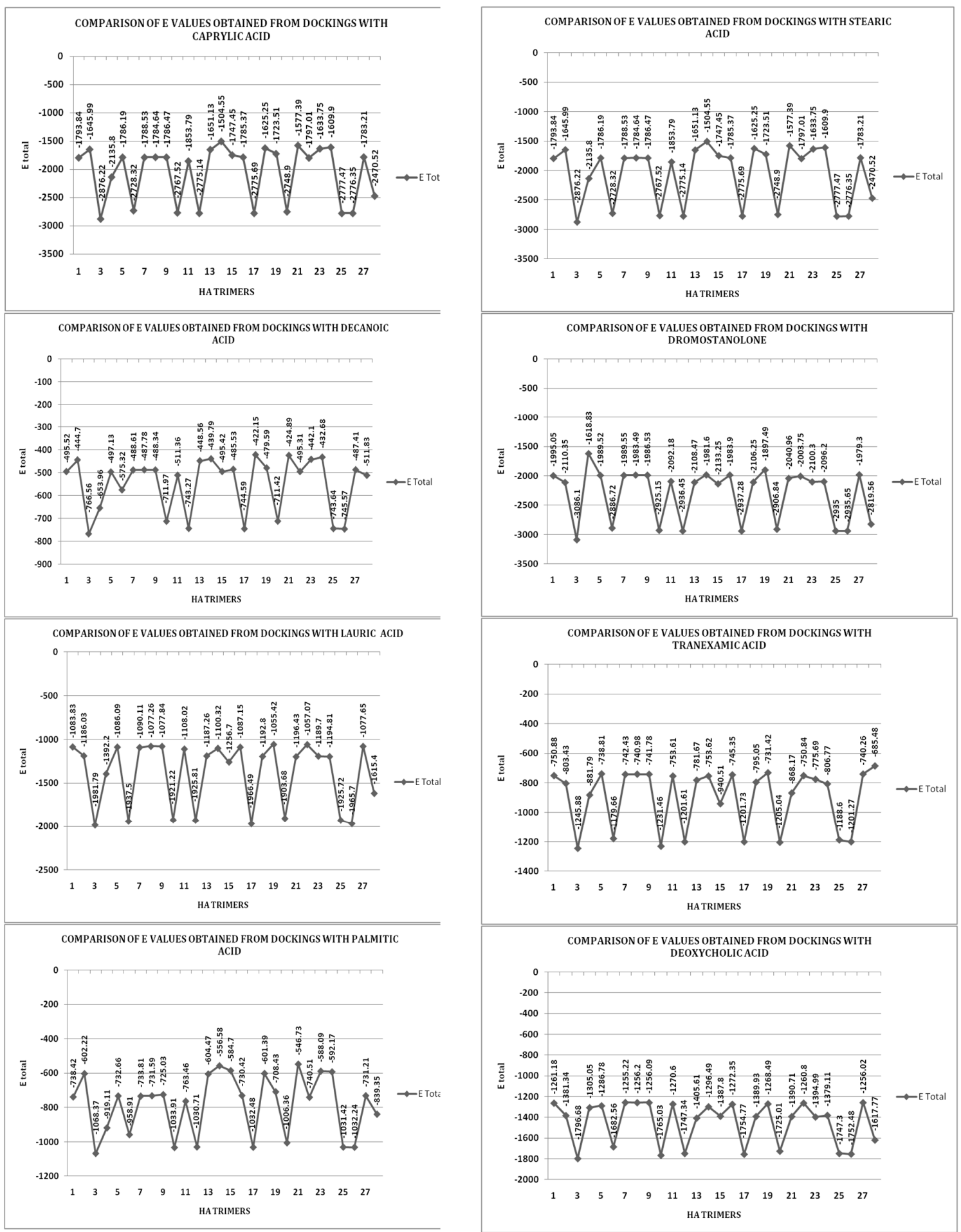


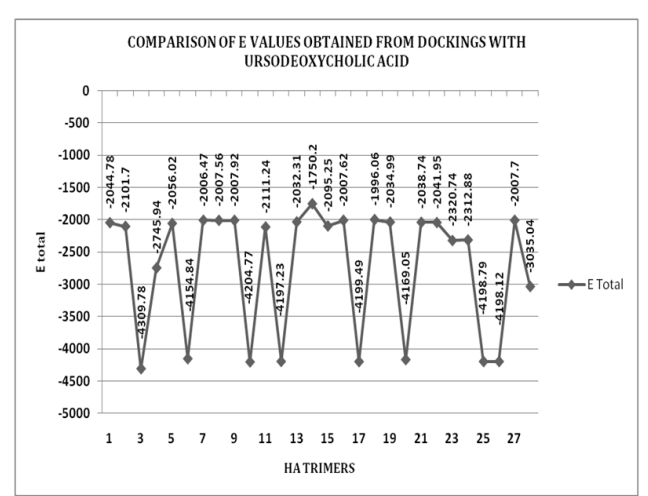

\section{ANALYSIS OF DOCKINGS}

Analysis of dockings revealed that the interactions of the three herbal inhibitors menthol, eucalyptol and ormosinine with the wild type and mutant haemagglutinins, are of a strong nature, whereas, the interactions of docosanol are extremely poor. The fatty acid inhibitors, stearic acid, palmitic acid and lauric acid have interacted strongly, whereas, caprylic acid and decanoic acid have shown moderate interactions. Dromostanolone, tranexamic acid, deoxycholic acid and ursodeoxycholic acid have exhibited very strong interactions with the mutant and wild type trimers.

The mutations also probably affect the inhibitorbinding site. This can be traced from the fact that, certain mutants, inspite of carrying entirely different mutations at completely different positions, has interacted very similarly with the different inhibitors. For instance, mutants $3,6,10,12,17,20,25$ and 26 carry entirely different mutations at different locations, and they have consistently exhibited a striking similarity in their interactions with the inhibitors. Interestingly, these eight mutants have also exhibited persistent strong interactions with the inhibitors (except docosanol and decanoic acid; in the former case, the interactions are of a poor nature; in the latter case, the interactions being of a moderate nature). The interactions of menthol, eucalyptol, ormosinine, caprylic acid, palmitic acid, dromostanolone, deoxycholic acid, ursodeoxycholic acid with the six mutants 1, 16, 19, 22 and 27, also reflects a similar incidence. Once again, such a similarity of interactions is observed in case of the mutants 2, 13, 14, 18, 21, 23 and 24, with six of the inhibitors, namely, menthol, eucalyptol, ormosinine, dromostanolone, deoxycholic acid and ursodeoxycholic acid.

Certain residues of the haemagglutinin have been used more frequently during the interactions with the inhibitors. Asn 96, Asp 97, Glu 227, residues 318-321 of chain A; Glu 11, residues 12-15 of chain $B$ are such residues. Some less frequently encountered residues include Tyr 7 , His 180 , Val 210, Glu 212 of chain A. The Asp 19, Gly 20, Glu 150 forms other such residues. Residues lying roughly in and around $150-160$ of chain B, serve as binding residues for some of the interactions of menthol, eucalyptol, ormosinine, palmitic acid, caprylic acid, deoxycholic acid and deoxycholic acid. (Refer supplementary: Table 3(a-m) for binding residues).

The glutamate at position 11 of the fusion peptide in chain $B$ has been reported to be responsible for promoting fusion of the haemagglutinin with the host receptors (Su et al., 2007). Some of the inhibitors in the present study, namely, menthol, eucalyptol, caprylic acid, decanoic acid, lauric acid, palmitic acid, stearic acid, dromostanolone, deoxycholic acid and ursodeoxycholic acid, have interacted with this very glutamate at position 11 of some of the mutants (refer supplementary: Table 3 for the binding residues). Since these inhibitors are binding to a residue that has been reported to be essential for haemagglutinin-host receptor fusion, hence, once these inhibitors have passed the clinical testing phase, they could act as potent inhibitors of H5N1.

\section{CONCLUSION}

From the present study, it is possible to conclude that the three herbal inhibitors menthol, eucalyptol and ormosinine might serve as able inhibitors of the H5 haemagglutinin, owing to their strong interactions with the latter. Menthol and eucalyptol also have an added advantage, owing to their binding to a site (the glutamate 11) that is essential for haemagglutinin fusion to host cell receptors. Except decanoic acid, all the fatty acid inhibitors have strong interactions. Tranexamic acid, dromostanolone and the bile acids have also shown interactions of a very string nature, and can be studied for usage as probable inhibitors of $\mathrm{H} 5$ in the future. It is also evident that mutations in the $\mathrm{H} 5$ are probably affecting inhibitor binding.

\section{REFERENCES}

[1] Anwar T., Lal, S.K. and Khan A.U. (2006) In Silico Biology, 6, 0015.

[2] Chen et al. (2010) Acta Crystallographica D66:12-21.

[3] Davis et al. (2007) Nucleic Acids Research 35:W375-W383.

[4] Wishart D.S., Knox C., Guo A.C., Shrivastava S., Hassanali M., Stothard P., Chang Z., Woolsey J. (2006) Nucleic Acids Res. 34 (Database issue):D668-72. PMID: 16381955

[5] Wishart D.S., Knox C., Guo A.C., Cheng D., Shrivastava S., Tzur D., Gautam B., Hassanali M. (2008) Nucleic Acids Res. 36(Database issue):D901-6. PMID: 18048412

[6] Durrer P., Galli C., Hoenke S., Corti C., Glück R., Vorherr T. and Brunner J. (1996) The Journal of Biological Chemistry, 271, 13417-13421. 
[7] Horimoto T. and Kawaoka Y. (2001) Clinical Microbiology Reviews, 14(1), 129149.

[8] Jongkon N., Mokmak W., Chuakheaw D., Shaw J., Tongsima S. and Sangma C. (2009) BMC Genomics, 10(Suppl 3):S24.

[9] Katz D. H., Marcellett J. F., Khali M. H., Pope L. E. and Katz L. R. (1991) Proceedings of National Academy of Science, USA, 88, 10825-10829.

[10] http://www.molinspiration.com

[11] http://cactus.nci.nih.gov/translate/

[12] Qi X., Li X., Rider P., Fan W., Gu H., Xu L., Yang Y., Lu S., Wang $H$. and Liu F. (2009) PLOS ONE, 4(3), e4682.

[13] Reed Mark L., Yen Hui-Ling, DuBois Rebecca M., Bridges Olga A., Salomon R., Webster R.G. and Charles J. Russell (2009) Journal of Virology, 83(8), 35683580.

[14] Rohm C., Zhou N., Suss J., Mackenzie J. and Webster R.G. (1996) Virology, 217: 508-516.

[15] Salahuddin, P. and Khan, A. U., Bioinformation. 2009; 4(1): 30-35.. 2009; 4(1): 30-35.

[16] Smirnov Y.A., Gitelman A.K., Govorkova, E.A., Lipatov A.S. and Kaverin N.V. (2004) Virus Research, 99(2), 205-208.

[17] Su Y., Zhu X., Wang Y., Wu M. and Tien P. (2007) Archives of Virology Volume 153 (2), 247-257.

[18] Veljkovic V., Veljkovic N., Muller C.P., Müller S., Glisic S., Perovic V. and Köhler H. (2009) BMC Structural Biology, 9,21.

[19] Voet D. and Voet J.G. (2004) Biochemistry, 3e, New York: John Wiley and Sons Inc.

[20] WHO (2004) World Health Organisation Global Alert and Response: http://www.who.int/csr/disease/influenza/preparedne ss2004_12_08/enl

[21] Yamada S., Suzuki Y., Suzuki T., Le M.Q., Nidom C.A., Sakai-Tagawa Y., Muramoto Y., Mutsumi Kiso M., Horimoto T., Shinya K., Sawada T., Kiso M., Usui T., Murata T., Lin Y., Hay A., Haire L.F., Stevens D.J., Russell R.J., Gamblin S.J., Skehel J.J. and Kawaoka Y. (2006), Nature, 444, 378-382.

[22] Zhou N. N., Senne D. A., Landgraf J. S., Swenson S. L., Erickson G., Rossow K., Lin Liu, Kyoung-jin Yoon, Scott Krauss, and Robert G. Webster (1999) Journal of Virology, 73(10), 8851-8856. 